Check for updates

Cite this: Chem. Sci., 2019, 10, 2385

๑ All publication charges for this article have been paid for by the Royal Society of Chemistry

Received 1st October 2018

Accepted 21st December 2018

DOI: $10.1039 / \mathrm{c} 8 \mathrm{sc} 04361 \mathrm{~b}$

rsc.li/chemical-science

\section{Minimalistic supramolecular proteoglycan mimics by co-assembly of aromatic peptide and carbohydrate amphiphiles $\uparrow$}

\author{
Alexandra Brito, (D) $\ddagger^{\mathrm{ab}}$ Yousef M. Abul-Haija, (D) $\$ \S^{\mathrm{c}}$ Diana Soares da Costa, (D) ab \\ Ramon Novoa-Carballal, (iD ab Rui L. Reis, (iD abd Rein V. Ulijn, (D) *efg \\ Ricardo A. Pires (iD *abd and Iva Pashkuleva (D) *ab
}

\begin{abstract}
We report the co-assembly of aromatic carbohydrate and dipeptide amphiphiles under physiological conditions as a strategy to generate minimalistic proteoglycan mimics. The resulting nanofibers present a structural, fluorenylmethoxycarbonyl-diphenylalanine (Fmoc-FF) core and a functional carbohydrate (Fmoc-glucosamine-6-sulfate or -phosphate) shell. The size, degree of bundling and mechanical properties of the assembled structures depend on the chemical nature of the carbohydrate amphiphile used. In cell culture medium, these nanofibers can further organize into supramolecular hydrogels. We demonstrate that, similar to proteoglycans, the assembled gels prolong the stability of growth factors and preserve the viability of cultured cells. Our results demonstrate that this approach can be applied to the design of extracellular matrix (ECM) substitutes for future regenerative therapies.
\end{abstract}

\section{Introduction}

Molecular self-assembly is a versatile bottom-up approach for the design and synthesis of functional biomaterials. ${ }^{1-5}$ The main advantage of this approach is the possibility to create structural and functional biomimetics with simple and systemically customizable compositions..$^{3-5}$ As an example, bioactive peptide amphiphiles (PA) of varying complexity have been used as simple functional analogues of various proteins. ${ }^{2-4}$

${ }^{a} 3 B$ 's Research Group, I3Bs - Research Institute on Biomaterials, Biodegradables and Biomimetics, University of Minho, Headquarters of the European Institute of Excellence on Tissue Engineering and Regenerative Medicine, AvePark, Parque de Ciência e Tecnologia, Zona Industrial da Gandra, 4805-017 Barco, Guimarães, Portugal.E-mail: pashkuleva@i3bs.uminho.pt; rpires@i3bs.uminho.pt

${ }^{b}$ ICVS/3Bs-PT Government Associate Laboratory, Braga/Guimarães, Portugal

${ }^{c}$ Department of Pure and Applied Chemistry, University of Strathclyde, Glasgow G1 $1 X L, U K$

${ }^{d}$ The Discoveries Centre for Regenerative and Precision Medicine, Headquarters at University of Minho, Avepark, 4805-017 Barco, Guimarães, Portugal

${ }^{e}$ Advanced Science Research Center (ASRC) at the Graduate Center, City University of New York (CUNY), 85 St Nicholas Terrace, New York, New York 10031, USA. E-mail: rein.ulijn@asrc.cuny.edu

${ }^{f}$ Department of Chemistry, Hunter College, City University of New York, 695 Park Avenue, New York 10065, USA

${ }^{g}$ PhD Programs in Biochemistry and Chemistry, The Graduate Center of the City University of New York, New York 10016, USA

$\dagger$ Electronic supplementary information (ESI) available: Synthetic procedures and characterization data. See DOI: 10.1039/c8sc04361b

$\$$ These authors contributed equally.

$\S$ Present address: WestCHEM, School of Chemistry, University of Glasgow, Glasgow G12 8QQ, UK
These building blocks can code specific biochemical information and transfer it to the surrounding (biological) environment. Glycosylation, the process of functional decoration of proteins with carbohydrate chains, is the most common posttranslational modification that diversifies the roles of proteins by instructing their folding and by activating specific recognition events and different signalling pathways. ${ }^{6}$ Attached carbohydrate chains can also have a protective role which contributes to the stability and activity of proteins. ${ }^{6,7}$ So far, these diverse roles of carbohydrates have received little attention, compared to peptides, in the design of molecular self-assembling blocks for the synthesis of functional supramolecular gels. ${ }^{8-14}$

Aiming to develop extracellular matrix (ECM) mimics, recent studies introduced the use of peptide amphiphiles (PAs) end-on functionalized with carbohydrate moieties. ${ }^{8-11}$ Upon assembly of such amphiphiles, the carbohydrate units are exposed on the surface of the assembled fibre and are able to participate in multivalent biospecific interactions. While this approach seems straightforward for generating supramolecular mimics of proteoglycans, the introduction of the carbohydrate unit is challenging in terms of PA synthesis and assembly as it may disturb the delicate balance of forces governing the process of self-organization. ${ }^{15,16}$

Multicomponent co-assembly offers a possibility to expand the chemical diversity and functional complexity of supramolecular systems without compromising the simplicity of the building blocks. ${ }^{17}$ The feasibility of this approach has been demonstrated by a combination of different PAs. ${ }^{15-18} \mathrm{We}$ hypothesized that a similar strategy can also be applied for coassembly of simple carbohydrate amphiphiles (CAs, 1) and their 


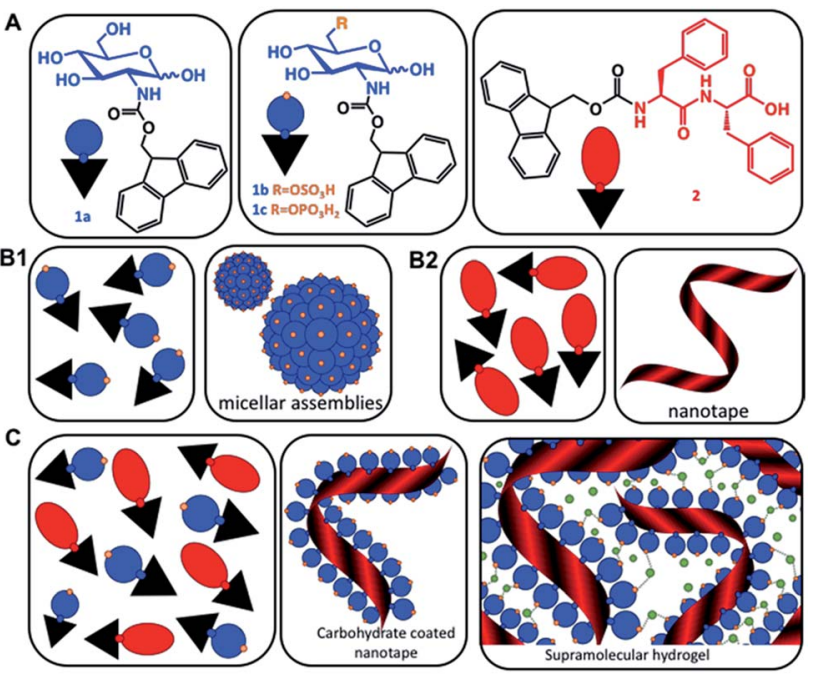

Scheme 1 (A) Chemical structures of the carbohydrate amphiphiles (1) and the peptide analogue Fmoc-FF (2). (B) Schematic representation of the assembly of the individual components (1) and (2) in water resulting in the formation of micelles and nanotapes, respectively. (C) Co-assembly of (1) and (2) to form carbohydrate functionalized peptide nanotapes and supramolecular hydrogels. Green dots represent divalent cations.

peptide analogues (2) and the result will be a synergistic modular system mimicking proteoglycans (Scheme 1).

The range of carbohydrates found in biological systems provide a rich library of water soluble, chiral building blocks. ${ }^{19}$ Simple carbohydrate amphiphiles (CAs) functionalized with aromatic moieties such as naphthalene and fluorenylmethoxycarbonyl (Fmoc) have been recently developed and shown to form self-assembled structures. ${ }^{20-22}$ However, the amphiphiles bearing the naphthalene unit do not form gels and the ones functionalized with Fmoc require a heating-cooling cycle or a specific enzymatic stimulus to enable the formation of gels under physiological conditions. ${ }^{20,21}$

Herein, we describe bi-component systems, in which fluorenylmethoxycarbonyl-diphenylalanine (Fmoc-FF) provides the structural component, while Fmoc-carbohydrate is the solvent-exposed functional element, which can be systematically exchanged (Scheme 1). We demonstrate that these modular materials combine the simplicity of small molecules with the versatility and biofunctionality of glycans and proteins, respectively.

\section{Results and discussion}

Self-assembly of aromatic carbohydrate amphiphiles (1) and Fmoc-FF (2)

The amphiphiles Fmoc-glucosamine-6-sulfate (GlcN6S, 1b) and Fmoc-glucosamine-6-phosphate (Fmoc-GlcN6P, 1c) were obtained from the respective glucosamines in a single-step reaction using an excess of Fmoc chloride (details on the synthesis and chemical characterization of the amphiphiles are provided in the ESI, Fig. S1-S13†).
Fmoc-GlcN (1a) is not soluble in water at room temperature. Upon temperature rising $\left(80^{\circ} \mathrm{C}\right)$, it dissolves and organizes spontaneously into nanofibers that, at room temperature, form a highly hydrated scaffold (Fig. 1A). ${ }^{20}$ On the other hand, FmocFF (2) dissolves in water only at basic $\mathrm{pH}(\sim 11)$ and selfassembles upon $\mathrm{pH}$ decrease. ${ }^{23-25}$ The assembly of 2 is controlled by peptidic hydrogen bonds that result in the formation of $\beta$-sheet-like structures that are further stabilized by aromatic $\pi-\pi$ interactions, whilst the organization of $1 \mathrm{a}$ is driven by a combination of $\mathrm{CH}-\pi$ and $\pi-\pi$ (T-shape) stacking interactions..$^{20,24,26}$

We compared the properties of the formed assemblies by AFM (Fig. 1B and C, black outlines) and found that the fibres have a similar size but their Young's moduli are significantly different: the PA 2 assembles in very rigid fibres ( $16 \mathrm{GPa})$ that have a 4 -fold higher modulus than the ones obtained from the carbohydrate analogue 1a $(\sim 4 \mathrm{GPa})$.

A
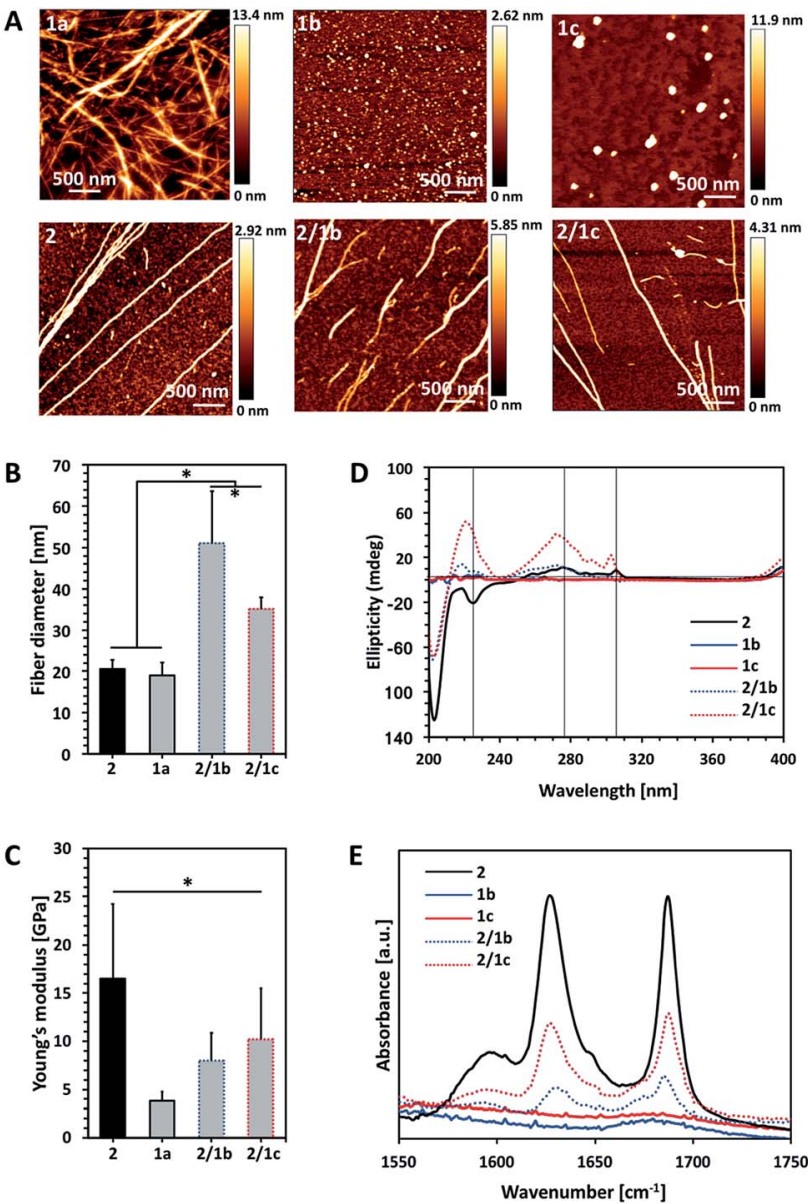

Fig. 1 Characterization of single $(10 \mathrm{mM})$ and bi-component (ratio PA : CA 2 : 1) assemblies: (A) AFM images; (B) fibre diameter and (C) Young's modulus of the assembled fibres measured by AFM; (D) CD spectra of the PA Fmoc-FF (2), its CA analogues Fmoc-GlcN6S (1b) and Fmoc-GlcN6P (1c), and the respective co-assemblies; (E) FTIR spectra of the precursor amphiphiles and their co-assemblies. Statistically significant differences $(p<0.01)$ are marked with (*). Of note, FmocGlcN (1a, $10 \mathrm{mM}$ ) was dissolved at $80^{\circ} \mathrm{C}$ and the solution gels upon its cooling to room temperature and thus, AFM analysis was performed on the gel. 
The co-assembly of $\mathbf{2}$ and $\mathbf{1 a}$ is challenging as both compounds have limited solubility in water and dissolve under different conditions. We functionalized 1a with polar groups aiming to improve the solubility and to impart relevant functionality. Sulphate groups (1) were chosen because of their biological relevance: most ECM glycosaminoglycans are sulphated and they interact specifically with other biological entities (involved in cell-cell and cell-ECM interactions) depending on their sulphation patterns. ${ }^{6}$ On the other hand, carbohydrates internalized by cells are activated via their functionalization with phosphate groups and thus, we have also chosen this functionality (1c). As expected, incorporation of these functional groups resulted in improved solubility and affected the assembly of Fmoc-GlcN6S (1b) and Fmoc-GlcN6P (1c): the introduced polar, negatively charged groups cause electrostatic repulsion between the modified $\mathrm{CA}$, thus disturbing the unidirectional assembly observed for 1a. Such Coulomb forces favour self-assembled morphologies with a high surface to volume ratio. Indeed, $\mathbf{1 b}$ and $\mathbf{1 c}$ form micellar assemblies with negative zeta potentials $(-112.0 \pm 23.7 \mathrm{mV}$ for $\mathbf{1 b}$ and $-74.4 \pm 5.3 \mathrm{mV}$ for 1c), indicating that the sulphate group of $\mathbf{1 b}$ and the phosphate group of 1c are displayed on the surface of the micelles formed in water (Fig. 1A). Of note, the micelles of 1c are bigger than the ones assembled from $\mathbf{1 b}$ due to the different Coulomb forces (Fig. S17†). The circular dichroism (CD) analysis supports the formation of micellar assemblies: no signals were observed in the CD spectra of $\mathbf{1 b}$ and $\mathbf{1 c}$ (Fig. 1D).

Co-assembly of $\mathbf{2}$ with either $\mathbf{1 b}$ or $\mathbf{1 c}$ was performed in water at room temperature. The respective amphiphiles were dissolved at basic $\mathrm{pH}(\sim 11)$ at different ratios (i.e. PA : CA of $2: 0.5$, $2: 1,2: 2$ ) but transparent solutions were observed only for the ratios $2: 0.5$ and $2: 1$ (Fig. $\mathrm{S} 14 \dagger$ ). The $\mathrm{pH}$ was then adjusted to 8 by drop-wise addition of $\mathrm{HCl}$. CD spectra of these solutions were recorded $24 \mathrm{~h}$ after the $\mathrm{pH}$ adjustment and indicated the formation of assemblies that clearly differ from 2 (Fig. 1D and $\left.\mathrm{S} 18^{\dagger}\right)$. The peak at $204 \mathrm{~nm}$ is associated with the $\pi-\pi^{*}$ transition and is clearly observed for all systems that contain 2 . Formation of a superhelical arrangement ( $n-\pi^{*}$ transition) is shown by the signal at $225 \mathrm{~nm}$ in the spectrum of $2 .^{26,27}$ A blue shift of this peak, observed in the mixed systems $(217 \mathrm{~nm}$ for $2 / 1 \mathbf{b}$ and $220 \mathrm{~nm}$ for $2 / \mathbf{1 c}$ ), indicates changes in the superhelical structure. ${ }^{28,29}$ This shift was concomitant with polarity changes upon addition of CAs and increased intensity especially for $\mathbf{2 / 1}$, indicating co-assembly of the CAs with 2 . Signals associated with the $\pi-\pi *$ transition of the Fmoc groups (the signal with the maximum at $278 \mathrm{~nm}$ and the peak at $306 \mathrm{~nm}$ in the spectrum of 2, Fig. 1D) are also blue-shifted in the spectra of the mixed systems confirming co-assembly. ${ }^{27}$

TEM and AFM characterization corroborated the CD and fluorescence data. The co-assembled fibres differ from the single component fibres in several aspects: both 2/1b and 2/1c fibres are bundled (and appear branched), thicker and less stiff than the Fmoc-FF fibres (Fig. 1A-C and S16, S20 $\dagger$ ). Furthermore, the co-assembled fibres have higher Young's moduli compared to the single component CA (1a) fibres, i.e. the co-assemblies have intermediate mechanical properties when compared with the PA and CA single component fibres. FTIR spectroscopy was also performed to characterize the co-assemblies (Fig. 1E). ${ }^{30,31}$ Absorptions at 1625 and $1687 \mathrm{~cm}^{-1}$ are associated with the formation of $\mathrm{H}$-bonding by Fmoc-peptide amide and carbamate groups. ${ }^{32}$ As expected, these peaks are missing in the spectra of CA (1) and 1c) solutions. On the other hand, the amide I peaks are clearly observed for the co-assembled systems (2/1b and 2/1c) demonstrating that the $\beta$-sheet structure remains intact in the presence of the CA although the lower intensity of these peaks suggests that a degree of intercalation might have occurred.

Previously, different models for assembly of two-component systems have been proposed. ${ }^{16,17,31}$ Our results (AFM; CD and FTIR spectra) indicate that $\mathbf{2}$ co-assembles with $\mathbf{1 b}$ and $\mathbf{1 c}$ into core-shell nanofibers with the carbohydrate moieties exposed on the surface. Such co-assembly would involve intercalation between the Fmoc moieties of the CA and PA on the formed fibre surface (Scheme 1B), thus shielding the hydrophobic Fmoc while exposing the carbohydrate unit. ${ }^{31}$ We studied the surface charge of the formed nanofibers in order to confirm this organization (Table S1 $\dagger$ ). The value determined for 2 was $-0.04 \mathrm{mV}$, which agrees with our previous work showing that the apparent $\mathrm{p} K_{\mathrm{a}}$ of the terminal carboxylic acid in 2 is substantially shifted due to the favoured self-assembly of the uncharged form. ${ }^{23}$ The co-assembled systems $\mathbf{2 / 1}$ b and $\mathbf{2 / 1}$ c have negative zeta potentials, $-56.0 \pm 1.5 \mathrm{mV}$ and $-55.4 \pm 6.1 \mathrm{mV}$ respectively. As expected, the absolute values are smaller than the ones of the precursor CAs due to the lower density of the functional groups on the co-assembled fibres as compared with the single component micelles. These results further confirm our hypothesis for co-assembly resulting in the formation of systems with a peptide-based core and carbohydrate shell.

\section{Characterization of the supramolecular gels}

Upon contact with cell culture medium ( $\mathrm{pH} 7.4$ ), the PA 2 and its co-assemblies with the CAs form self-sustained macroscopic gels (Fig. 2A).

We used an AFM to analyse the gels and observed a typical entangled fibrous network for all studied compositions (Fig. 2B and $\mathrm{S} 21 \dagger$ ). However, two major differences can be observed in the AFM images: fibres of 2 are thinner and more entangled compared to the ones generated by co-assembly (2/1b and $2 / \mathbf{1 c})$. These differences are also reflected in the viscoelastic properties of the gels: mixed systems form more robust gels in all studied media (Fig. 2C and S23†). Previous studies have demonstrated that gelation of similar PA systems can be either $\mathrm{pH}$ - or salttriggered via the formation of salt bridges between the anions exposed on the fibre surface (Scheme 1C). ${ }^{33,34}$ Our rheological studies confirmed that these stabilizing interactions are also at play in our current system: upon reduction of the $\mathrm{pH}$ by adding Dulbecco's Phosphate-Buffered Saline (DPBS, pH 7.4, only monovalent cations), we observed the formation of soft gels. The moduli of the formed gels increased significantly in the presence of divalent $\mathrm{Ca}^{2+}$ cations (Fig. 2C, DPBS $+\mathrm{CaCl}_{2}$ ). This difference depends on the anions exposed on the fibre surface and we observed the following order $\mathrm{HPO}_{4}{ }^{2-} / \mathrm{H}_{2} \mathrm{PO}_{4}{ }^{-}(2 / 1 \mathrm{c} 3.3-$ fold increase $)>\mathrm{SO}_{4}{ }^{2-}(2 / 1 \mathbf{b}, 3$-fold increase $)>\mathrm{COO}^{-}(2,2.7$-fold 
A
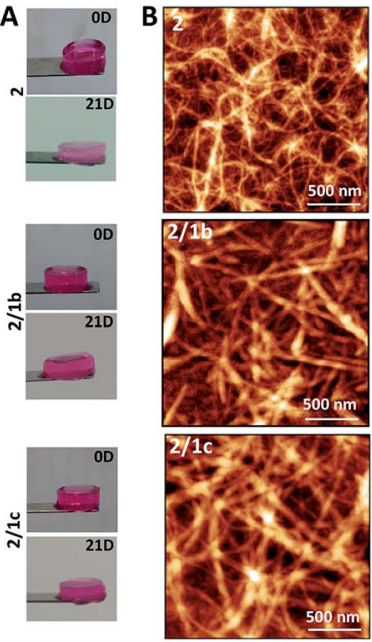

\section{C}

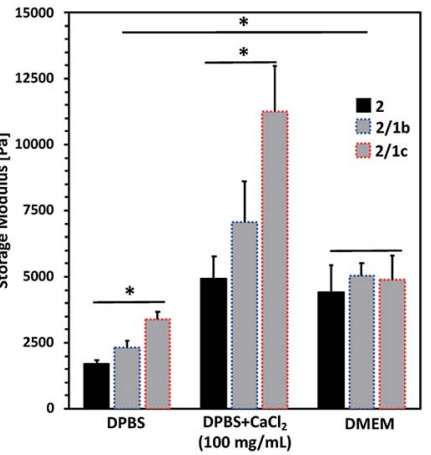

Fig. 2 Characterization of the formed gels: (A) optical images showing the macroscopic integrity of the gels after formation in cell culture medium (DMEM) at $37^{\circ} \mathrm{C}$ (OD) and after immersion for 21 days under the same conditions (21D); (B) tangled nanofibrous structures observed using an AFM; (C) mechanical properties of the gels determined by rheology. DPBS: Dulbecco's Phosphate-Buffered Saline (with no $\mathrm{Ca}^{2+}$ and $\mathrm{Mg}^{2+}$ ); DMEM: Dulbecco's Modified Eagle's Medium; DMEM was supplemented with $10 \%$ fetal bovine serum.

increase). These results confirmed that the carbohydrate units are exposed on the fibre surface.

After confirming the exposure of the carbohydrate units on the fibre surface and their ability to form gels under physiological conditions, we evaluated the bioactivity of the generated co-assemblies by their ability to interact specifically with proteins. We designed two experimental setups to evaluate two fundamental roles of proteoglycans in the ECM: the ability of the assembled gels to protect growth factors and to host cells.

\section{Interactions of the supramolecular gels with basic fibroblast growth factors (FGF-2)}

Basic fibroblast growth factor (FGF-2) is a glycoprotein that has a pleiotropic effect in different tissues and organs: it is crucial for processes such as angiogenesis, development, wound healing, cell survival and differentiation, among others. ${ }^{35,36}$ It is also known for its thermal instability: it loses most of its activity after $24 \mathrm{~h}$ of incubation at $37{ }^{\circ} \mathrm{C}$ (Fig. S24 $\dagger$ ). ${ }^{37}$ In its native environment - the pericellular space - FGF-2 interacts specifically with cell surface proteoglycans, which protect it from degradation and aggregation. ${ }^{6}$ Electrostatic interactions and hydrogen bonding are the main driving forces for the formation of proteoglycan-FGF-2 complexes. ${ }^{36,38}$ Proteoglycan charge density is crucial for these interactions as basic amino acid residues in the growth factor, usually ordered in Cardin-Weintraub sequences, are the main contributors to the formation of the complexes. ${ }^{6,38}$ Indeed, addition of heparin (the most negatively charged natural biomolecule) or heparan sulphate to the FGF-2 incubation media prolongs its activity. ${ }^{37,39,40}$ We therefore tested the developed systems for their capacity to protect FGF-2 and preserve its bioactivity under physiological conditions (Fig. 3). ${ }^{9}$
A 2_0D

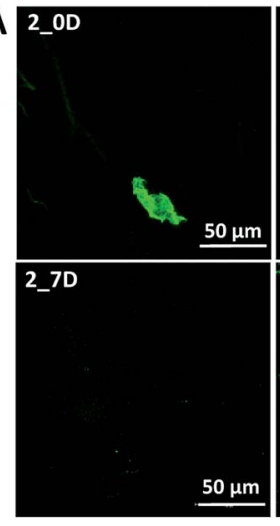

2/1b_OD

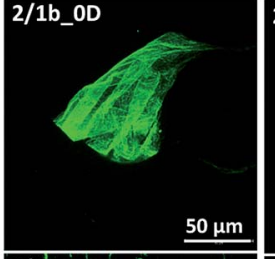

2/16_7D
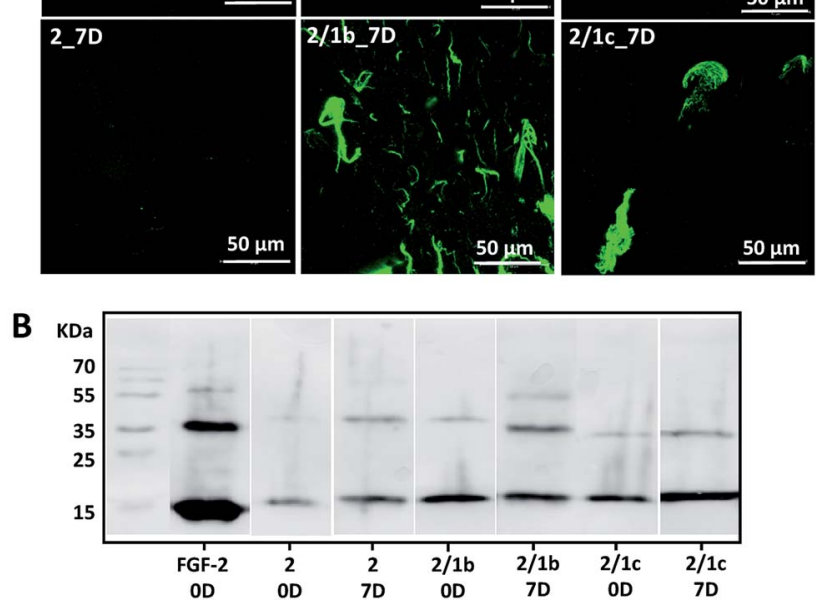

Fig. 3 Interaction of one- and bi-component supramolecular hydrogels with FGF-2: (A) bioactive FGF-2 (green) distribution and stability within the gels demonstrated for 0 and 7 days by confocal imaging and immunostaining. Of note, FGF-2 was not homogenised intentionally to facilitate the visualization of the growth factor by fluorescent microscopy; (B) SDS-PAGE analysis of FGF-2 entrapped (OD) and preserved in the hydrogel after 7 days.

We added the growth factor into the gels (details are provided in the ESI $\dagger$ ) and the successful encapsulation was confirmed by immunostaining and SDS-PAGE (Fig. 3, 0D). Of note, immunostaining allows visualisation only of active FGF-2 as it is based on the recognition of the growth factor by a specific antibody with a fluorescent tag. The gels with the encapsulated growth factor were maintained under standard cell culture conditions (DMEM at $37{ }^{\circ} \mathrm{C}$ ) for 7 days and then characterised again (Fig. 3, 7D). Our results demonstrated that mixed systems (2/1b and 2/1c) protect better the FGF-2 from degradation when compared to the non-functionalized peptide nanofibers (2): stronger co-localization (Fig. 3A) and more protein (Fig. 3B) are visible for the FGF-2 loaded in the co-assembled gels after 7 days. Moreover, ELISA tests did not show any release of the growth factor to the surrounding media for this period of time. These results indicate that FGF-2 is simply entrapped in the Fmoc-FF gel and thus, attainable for degradation (although slower as compared with free FGF-2). On the other hand, in the mixed systems, FGF-2 interacts with the carbohydrates exposed on the fiber surface, thus copycatting the natural proteoglycan protection mechanism.

\section{Cytotoxicity of the supramolecular gels}

Next, we tested the ability of the gels to support viable cells. Initially, we used L929 cells that are recommended for standard in vitro cytotoxicity tests. Our results demonstrate that cells remain viable either when they are seeded on top of the gels 

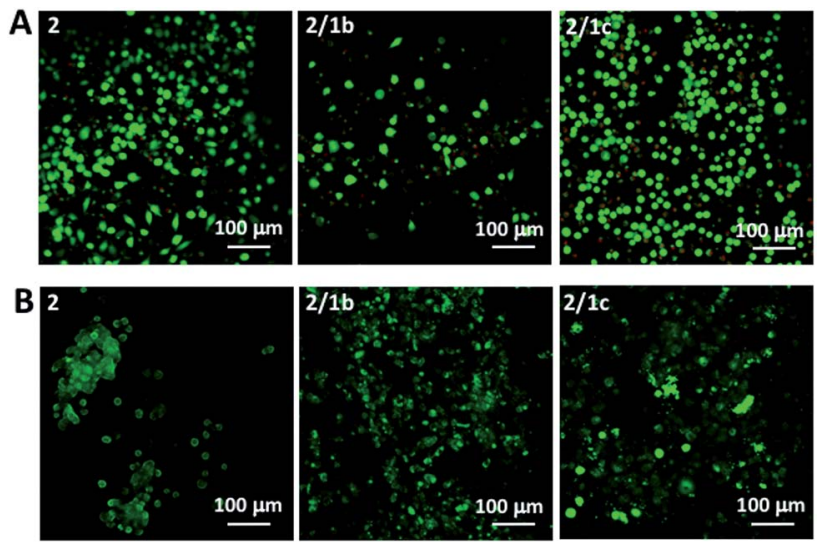

Fig. 4 Live/dead assays for L929 cultured (A) on top of the gels or (B) encapsulated within the gels for $48 \mathrm{~h}$. Dulbecco's Modified Eagle's Medium (DMEM) was used for these tests.

(Fig. 4A and S27 $\dagger$ ) or when they are encapsulated inside the gels (Fig. 4B and S27†). Chondrogenic cell line ATDC5 (Fig. S25†) and epithelial HeLa cells (Fig. S26†) were also tested with the assembled materials to prove their versatility. In all the cases, we observed a majority of viable cells (Fig. S27†). The culture of these cell lines requires the use of media supplemented with proteins $(10 \%$ fetal bovine serum, FBS $)$ that can affect the mechanical properties of the gels..$^{41}$ Our results demonstrated that indeed the presence of proteins affects significantly the storage moduli of the gels: when DPBS supplemented with $\mathrm{CaCl}_{2}$ was replaced with DMEM (the same $\mathrm{Ca}^{2+}$ concentration) supplemented with FBS we observed a significant decrease of the storage moduli, especially for the co-assembled systems (Fig. 2C and S23†). Of note, the substitution of DMEM with DMEM/F12 does not induce any significant change in the mechanical properties of the gels (Fig. S23†). These data suggest that the proteins from the medium interact with the mixed systems and disrupt partially the ionic crosslinking bridges between different nanofiber segments. As the mechanical properties of the assembled gels under cell culture conditions are very similar, we suggest that the observed different number of adherent/encapsulated cells is due to the different chemistry of the generated supramolecular systems and their specific interactions with the cultured cells.

\section{Conclusions}

We have successfully designed and synthesized supramolecular, functional glycoprotein mimics by co-assembly of simple building blocks. In addition to the simplicity of the building blocks, the proposed approach has several other advantages: the assembly is carried out under physiological conditions, and the formed hydrogels are stable, non-cytotoxic and biofunctional. We also demonstrated that by changing the chemical design in a systematic way we are able to control the biofunctionality and mechanical properties of the supramolecular gels - features that are of key importance in the design of materials for biomedical and tissue engineering applications.

\section{Conflicts of interest}

Rein V. Ulijn is a director of Biogelx, Ltd, an organization with a financial interest in the subject matter and materials (FmocFF) discussed in this publication.

\section{Acknowledgements}

We acknowledge the EU's H2020 and FP7 framework programmes (Forecast 668983; CHEM2NATURE 692333; THE DISCOVERIES CTR 739572; ERC AdG ComplexiTE 321266) and the Portuguese FCT (IF/00032/2013; BD/113794/2015; BPD/ 85790/2012; M-ERA-NET2/0001/2016 - INCIPIT; ENMed/001/ 2015 - CytoNanoHeal). We thank P. Frederix for his help in the FTIR measurements and M. Mullin for her help in the TEM imaging.

\section{References}

1 D. B. Amabilino, D. K. Smith and J. W. Steed, Chem. Soc. Rev., 2017, 46, 2404-2420.

2 J. Boekhoven and S. I. Stupp, Adv. Mater., 2014, 26, 16421659.

3 X. W. Du, J. Zhou, J. F. Shi and B. Xu, Chem. Rev., 2015, 115, 13165-13307.

4 K. E. Inostroza-Brito, E. Collin, O. Siton-Mendelson, K. H. Smith, A. Monge-Marcet, D. S. Ferreira, R. P. Rodriguez, M. Alonso, J. C. Rodriguez-Cabello, R. L. Reis, F. Sagues, L. Botto, R. Bitton, H. S. Azevedo and A. Mata, Nat. Chem., 2015, 7, 897-904.

5 M. J. Webber, E. A. Appel, E. W. Meijer and R. Langer, Nat. Mater., 2016, 15, 13-26.

6 D. S. Costa, R. L. Reis and I. Pashkuleva, Annu. Rev. Biomed. Eng., 2017, 19, 1-26.

7 H. S. Azevedo and I. Pashkuleva, Adv. Drug Delivery Rev., 2015, 94, 63-76.

8 X. W. Du, J. Zhou, O. Guvench, F. O. Sangiorgi, X. M. Li, N. Zhou and B. Xu, Bioconjugate Chem., 2014, 25, 1031-1035.

9 S. S. Lee, T. Fyrner, F. Chen, Z. Álvarez, E. Sleep, D. S. Chun, J. A. Weiner, R. W. Cook, R. D. Freshman, M. S. Schallmo, K. M. Katchko, A. D. Schneider, J. T. Smith, C. Yun, G. Singh, S. Z. Hashmi, M. T. McClendon, Z. Yu, S. R. Stock, W. K. Hsu, E. L. Hsu and S. I. Stupp, Nat. Nanotechnol., 2017, 12, 821-829.

10 B. S. Kim, D. J. Hong, J. Bae and M. Lee, J. Am. Chem. Soc., 2005, 127, 16333-16337.

11 J. Zhou, X. W. Du, X. Y. Chen and B. Xu, Biochemistry, 2018, 57, 4867-4879.

12 Y. B. Lim, S. Park, E. Lee, H. Jeong, J. H. Ryu and M. S. Lee, Biomacromolecules, 2007, 8, 1404-1408.

13 J. Liu, Z. L. Sun, Y. Q. Yuan, X. Tian, X. Liu, G. X. Duan, Y. G. Yang, L. Yuan, H. C. Lin and X. M. Li, ACS Appl. Mater. Interfaces, 2016, 8, 6917-6924.

14 D. Yuan, J. Shi, X. Du, N. Zhou and B. Xu, J. Am. Chem. Soc., 2015, 137, 10092-10095.

15 J. Raeburn and D. J. Adams, Chem. Commun., 2015, 51, 51705180. 
16 L. E. Buerkle and S. J. Rowan, Chem. Soc. Rev., 2012, 41, 6089-6102.

17 P. Makam and E. Gazit, Chem. Soc. Rev., 2018, 47, 3406-3420.

18 Y. M. Abul-Haija, G. G. Scott, J. K. Sahoo, T. Tuttle and R. V. Ulijn, Chem. Commun., 2017, 53, 9562-9565.

19 M. Delbianco, P. Bharate, S. Varela-Aramburu and P. H. Seeberger, Chem. Rev., 2016, 116, 1693-1752.

20 L. S. Birchall, S. Roy, V. Jayawarna, M. Hughes, E. Irvine, G. T. Okorogheye, N. Saudi, E. De Santis, T. Tuttle, A. A. Edwards and R. V. Ulijn, Chem. Sci., 2011, 2, 1349-1355.

21 R. A. Pires, Y. M. Abul-Haija, D. S. Costa, R. Novoa-Carballal, R. L. Reis, R. V. Ulijn and I. Pashkuleva, J. Am. Chem. Soc., 2015, 137, 576-579.

22 Z. M. Yang, G. L. Liang, M. L. Ma, A. S. Abbah, W. W. Lu and B. Xu, Chem. Commun., 2007, 843-845, DOI: 10.1039/ b616563.

23 C. Tang, A. M. Smith, R. F. Collins, R. V. Ulijn and A. Saiani, Langmuir, 2009, 25, 9447-9453.

24 A. M. Smith, R. J. Williams, C. Tang, P. Coppo, R. F. Collins, M. L. Turner, A. Saiani and R. V. Ulijn, Adv. Mater., 2008, 20, 37-41.

25 A. Mahler, M. Reches, M. Rechter, S. Cohen and E. Gazit, Adv. Mater., 2006, 18, 1365-1370.

26 V. Jayawarna, M. Ali, T. A. Jowitt, A. E. Miller, A. Saiani, J. E. Gough and R. V. Ulijn, Adv. Mater., 2006, 18, 611-614.

27 Y. Zhang, H. W. Gu, Z. M. Yang and B. Xu, J. Am. Chem. Soc., 2003, 125, 13680-13681.
28 D. H. Chin, R. W. Woody, C. A. Rohl and R. L. Baldwin, Proc. Natl. Acad. Sci. U. S. A., 2002, 99, 15416-15421.

29 S. Mondal, L. Adler-Abramovich, A. Lampel, Y. Bram, S. Lipstman and E. Gazit, Nat. Commun., 2015, 6, 8615.

30 Y. M. Abul-Haija, S. Roy, P. W. Frederix, N. Javid, V. Jayawarna and R. V. Ulijn, Small, 2014, 10, 973-979.

31 S. Fleming, S. Debnath, P. W. J. M. Frederix, N. T. Hunt and R. V. Ulijn, Biomacromolecules, 2014, 15, 1171-1184.

32 S. Fleming, P. W. Frederix, I. Ramos Sasselli, N. T. Hunt, R. V. Ulijn and T. Tuttle, Langmuir, 2013, 29, 9510-9515.

33 L. Chen, G. Pont, K. Morris, G. Lotze, A. Squires, L. C. Serpell and D. J. Adams, Chem. Commun., 2011, 47, 12071-12073.

34 S. Roy, N. Javid, P. W. J. M. Frederix, D. A. Lamprou, A. J. Urquhart, N. T. Hunt, P. J. Halling and R. V. Ulijn, Chem.-Eur. J., 2012, 18, 11723-11731.

35 C. M. R. Perez, Z. Alvarez, F. Chen, T. Aytun and S. I. Stupp, ACS Biomater. Sci. Eng., 2017, 3, 2166-2175.

36 S. Amorim, R. A. Pires, D. S. da Costa, R. L. Reis and I. Pashkuleva, Langmuir, 2013, 29, 7983-7992.

37 G. Chen, D. R. Gulbranson, P. Z. Yu, Z. G. Hou and J. A. Thomson, Stem Cells, 2012, 30, 623-630.

38 D. M. Ornitz, BioEssays, 2000, 22, 108-112.

39 D. Gospodarowicz and J. Cheng, J. Cell. Physiol., 1986, 128, 475-484.

40 O. Saksela, D. Moscatelli, A. Sommer and D. B. Rifkin, J. Cell Biol., 1988, 107, 743-751.

41 N. Javid, S. Roy, M. Zelzer, Z. M. Yang, J. Sefcik and R. V. Ulijn, Biomacromolecules, 2013, 14, 4368-4376. 